Letters to the Editor should be typed double-spaced (including references) with conventional margins. The overall length is limited to five manuscript pages, including not more than one figure or one table.

\section{External Quality Control for Radloimmunoaseay of Urinary Albumin in Diabotic Subjects: A One-Year Experience}

\section{To the Editor:}

In diabetic populations, who have an increased prevalence of hypertension and renal disease, the assay of urinary albumin excretion (UAE) has assumed an important role in the prevention and monitoring of diabetic nephropathy and in the evaluation of cardiovascular risk (1-4). Because screening for microalbuminuria has become part of routine diabetes care and involves an increasing number of patients, a suitable and standardized method should be used for measuring albumin in urine. To meet these needs, one must know and try to minimize all analytical sources of variation and error. Although several immunological procedures (such as enzyme immunoassay, immunoturbidimetric assay, fluoroimmunoassay, and immunonephelometric assay) have been widely used for routine measurement of UAE, radioimmunoassay is generally considered the most reliable method $(5,6)$. Because external quality assessment (EQA) programs contribute to improving the analytical performances of participating laboratories through the comparison of their results with those produced by others, our laboratory, starting in March 1988, organized a national pilot EQA program for urinary albumin RIA to estimate the variability among and within laboratories in Italy as well as to check the possibility of improving, by an EQA survey, laboratory performance in measuring microalbuminuria. Here, we report results from the first year of this program.

The EQA scheme was based on the distribution of frozen pooled urine samples, followed by the computerized analysis of the returned results, as previously described (7-9). Participant laboratories assayed quality control (QC) samples by their routine procedures, returning results with the name of the method or kit used. After collected results had been processed by computer, summaries were prepared and sent back to each participant. Two EQA cycles have been accomplished (each cycle lasts $\sim 6$ months). Fifteen laboratories took part in the EQA program (11 in the first phase). Only two kits were used by the 15 participant laboratories: Albumin RIA 100, supplied by Pharmacia (Uppsala, Sweden; $n=8 ; n=6$ in the first phase), and H-Albumin, by Sclavo (Cinisello Balsamo, Milano, Italy; $n=7 ; n=5$ in the first phase).

We prepared 450 QC samples from 13 urine pools to be sent to participants in the two cycles. During the first one, we mailed out, in five dispatches, 165 QC specimens to 11 laboratories (Table 1). All these samples had been prepared from three urine pools (pools 1, 2, and 3) with low (consensus mean $=5.2 \pm 0.95 \mathrm{mg} / \mathrm{L}$ ), me$\operatorname{dium}(23.6 \pm 3.3 \mathrm{mg} / \mathrm{L})$, and high (59.3 $\pm 8.1 \mathrm{mg} / \mathrm{L}$ ) albumin concentrations. During the second cycle, we sent 285 QC vials derived from 10 urine pools: three pools had been set up by adding to a low-albumin pool weighed amounts of human albumin (pools 4, 5 , and 6), and four pools had been prepared by progressive dilution of a high-albumin pool (pools 7, 8, 9, and 10). These seven pools were prepared to better evaluate the accuracy of participating laboratories, because the total variability found in the first cycle was surprisingly large (CVs from 48\% to 66\%). The last three pools derived from urine samples with low (consensus mean $=3.9 \pm 1.3 \mathrm{mg} / \mathrm{L}$ ), medium (19.5 $\pm 2.9 \mathrm{mg} / \mathrm{L})$, and high (52.9 \pm 8.7 $\mathrm{mg} / \mathrm{L}$ ) albumin contents (pools 11,12 ,

Table 1. One-Factor Analysis of Variance of 145 Urinary Albumin Determinations in the First Cycle of the EQA Survey and of 175 Urinary Albumin Determinations in the Second Cyele

\begin{tabular}{|c|c|c|c|c|c|c|c|}
\hline \multirow[b]{2}{*}{ Urine pool } & \multirow[b]{2}{*}{$\begin{array}{c}\text { Aceanys, } \\
\text { n }\end{array}$} & \multirow[b]{2}{*}{$\begin{array}{l}\text { Great mean } \\
\quad \pm \text { SD }\end{array}$} & \multicolumn{3}{|c|}{ CV, $\%$} & \multicolumn{2}{|c|}{ Whthin-ktt CV, $\%$} \\
\hline & & & $\begin{array}{l}\text { Total } \\
\left(\mathrm{CV}_{\mathrm{T}}\right)^{\circ}\end{array}$ & 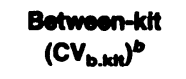 & $\begin{array}{l}\text { Whthin-kth } \\
\left(\mathrm{CV}_{\text {w.ton }}\right)^{\text {e.d }}\end{array}$ & $\begin{array}{l}\text { Bermeentlab } \\
\left(C V_{\text {bleat }}\right)^{\circ}\end{array}$ & 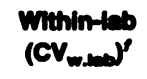 \\
\hline \multicolumn{8}{|c|}{ First phase (11 laboratories) } \\
\hline 1 (low) & 55 & $4.55 \pm 2.32$ & 51.0 & $34.6(46 \%)^{\circ}$ & $37.5(54 \%)^{n}$ & $28.2(57 \%)$ & $24.7(43 \%)$ \\
\hline 2 (medium) & 44 & $18.95 \pm 9.1$ & 48.0 & $30.4(40 \%)^{0}$ & $37.1(60 \%)^{n}$ & $29.9(64 \%)$ & 22.4 (36\%) \\
\hline 3 (high) & 46 & $38.6 \pm 24.3$ & 65.9 & $52.3(63 \%)^{0}$ & $40.2(37 \%)^{n}$ & $33.4(69 \%)$ & $22.3(31 \%)$ \\
\hline \multicolumn{8}{|c|}{ Second phase (15 laboratories) } \\
\hline 11 (low) & 56 & $3.42 \pm 1.66$ & 48.5 & $9.6(3.8 \%)$ & $47.5(96 \%)^{\prime}$ & $42.4(86 \%)$ & $16.9(14 \%)$ \\
\hline 12 (medium) & 60 & $20.9 \pm 2.9$ & 13.8 & $2.9(3.7 \%)$ & $13.5(96 \%)^{\prime}$ & $11.5(72 \%)$ & $7.1(28 \%)$ \\
\hline 13 (high) & 59 & $52.2 \pm 9.4$ & 18.1 & $4.8(7.2 \%)$ & $17.4(93 \%)^{\prime}$ & $14.9(75 \%)$ & $8.7(25 \%)$ \\
\hline \multicolumn{8}{|c|}{ 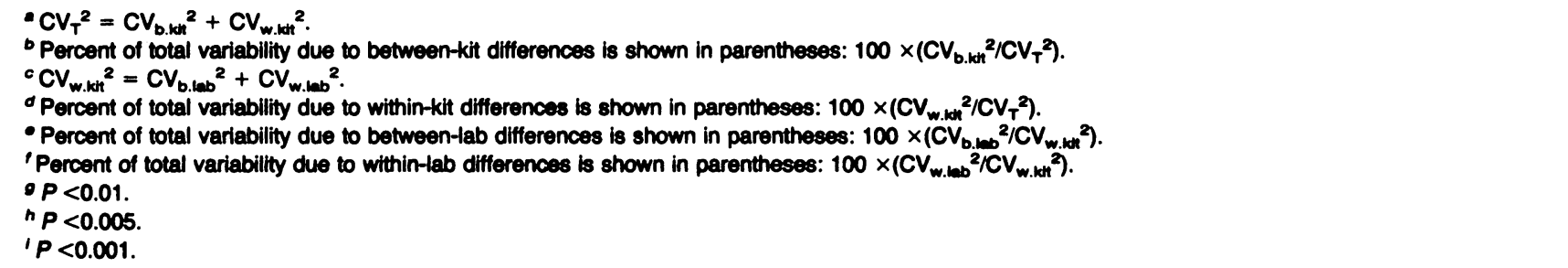 } \\
\hline
\end{tabular}


and 13; Table 1). All urine samples used for QC sample preparation were negative for bacterial growth and contained sodium axide $(\sim 2 \mathrm{~mL}$ of a 200 g/L solution per liter of urine). Prepared QC aliquots were frozen at $-20^{\circ} \mathrm{C}$ until sent to participants, packed in solid $\mathrm{CO}_{2}$ in polystyrene containers.

All results collected in each EQA cycle were used to compute $(7-9)$ the great or general mean (for each pool), the consensus mean (for each pool), laboratory bias, laboratory precision, and total variability or between-lab agreement (CV) and components of variability. We also calculated the outlier results by two statistical approaches, the target-value method and the Cochran-Grubbs test (10). For the target-value method, throughout each phase of the survey, we considered as outliers all the values that were $>2$ SD from the means of the RIA results provided by our laboratory (target values). During the first cycle of the EQA program, pools 1,2 , and 3 were tested 42,42 , and 28 times, respectively, by our laboratory in different experimental sessions. In the second phase, pools 11,12 , and 13 were measured 55,51 , and 47 times, respectively, in different sessions.

The consensus means calculated after deletion of outliers by the Cochran-Grubbs test were very similar to the means obtained after exclusion of outliers by the target method, except for QC pool 3. The sequential application of the Cochran-Grubbs test allowed the removal of three laboratories in the first phase (one for pool 2 and two for pool 3) and none in the second phase of EQA. A significant decrease of variability between the first and the second EQA phases was also confirmed by the reduction of outlier results as calculated by the targetvalue method: 62 of 145 results (42\%) of the first phase and 46 of 278 results (16.5\%; $P<0.001)$ of the second phase were $>2$ SD of the target values.

The total variability $\left(\mathrm{CV}_{\mathrm{T}}\right)$ of urine pools 1,2 , and 3 , sent during the first phase of EQA, was very large, ranging from $48 \%$ to $66 \%$. The within-kit and the between-kit components of variability were quite similar, thus indicating that both the imprecision of the two RIA kits and the between-kit difference were important causes of the high urinary albumin assay imprecision in that EQA phase (Table 1). In four laboratories, the mean bias showed a systematic deviation from the consensus mean, whereas in two other laboratories, the confidence limits of the mean appeared extremely wide. Results from these six laboratories accounted for the large number of outliers found in the first phase of the EQA program.

The analysis of the recovery and the dilution tests showed good results for 11 of 15 laboratories. Two laboratories had poor results for both the recovery (mean of the absolute values of the percent deviations from the expected values $>20 \%$ ) and the dilution tests (intercept significantly different from zero). Two other laboratories had poor results only for the recovery test. These four laboratories accounted for $>50 \%$ of the outliers. The total variability remained very large for pool 11 (48\%), but was substantially lower for pools 12 and 13 compared with $C_{T}$ observed for similar pools in the first EQA phase (pools 2 and 3, Table 1). Regardless of the albumin concentration, the between-kit component of the variability was lower in the second $(<10 \%)$ than in the first phase (30$50 \%)$ of the EQA; no differences were observed for results obtained with the two kits.

Laboratories with both accuracy and precision $>25 \%$ improved their performances substantially during the second phase of the EQA survey (4/11 vs $12 / 15$; chi-square $=5.10 ; P=0.024$ ) for assays of urine samples with medium and high albumin concentrations.

A prerequisite for the EQA program is the stability of EQA specimens throughout the study. We previously reported the stability over time of urine pools at $-20^{\circ} \mathrm{C}$ (as long as 6 months) (11) and after repeated freezing and thawing (as many as five times) (12). Stability of EQA samples was also confirmed by repeatedly measuring them in the reference laboratory throughout the two phases of the study. These data agree with others, indicating that urine samples are relatively stable for several months at $-20^{\circ} \mathrm{C}(6)$.

A very high variability (from $40 \%$ to $60 \%$ ) emerged from the first phase of the EQA program. This finding was surprising because RIA of urinary albumin is generally considered so robust, easy to perform, and reliable that it is suggested as the standard technique for UAE determination (5, 6). All participant laboratories measured UAE by only two commercial RIA kits. Conceivably, if laboratories using other RIAs or other methods (such as nephelometric or turbidimetric assay) had participated in the EQA scheme, the between-lab agreement would have been worse, because significant biases have been found among the different methods currently used for UAE $(5,6)$.

The total variability significantly improved in the second phase of the EQA survey, especially for albumin concentrations in the medium and high range. A large reduction in the between-kit component occurred too. Evidence consistent with ours was recently provided by a study (13) designed to check the existing variability in the measurement of albumin in urine among five laboratories using various immunoassays (enzyme immunoassay, immunoturbidimetric as say, fluorescent immunoassay, and zone immunoelectrophoresis assay). Results from this study indicated considerable variation both within and among laboratories; variability was equally attributable to imprecision and relative inaccuracy, being particularly high for materials with low albumin concentrations (13).

In conclusion, our data indicate that urinary albumin assays need to be adequately standardized, and an external QC of the assay is necessary even for laboratories that use a common assay procedure (such as RIA). Actually, the interlaboratory survey is the only way to quantitatively evaluate the performance of an RIA. The improvement in urinary albumin assay in the second EQA period was particularly relevant because it was obtained for those urinary albumin concentrations generally considered of high clinical usefulness for classifying diabetic people according to well-established risk categories for diabetic nephropathy and early cardiovascular mortality.

\section{References}

1. Viberti GC, Walker JD. Diabetic nephropathy: etiology and prevention. Diabetes Metab Rev 1988;4:147-62.

2. Mogensen CE. Microalbuminuria as a predictor of clinical diabetic nephropathy. Kidney Int 1987;31:673-89.

3. Bennett PH. "Microalbuminuria" and diabetes: a critique-assessment of urinary albumin excretion and its role in screening of diabetic nephropathy. Am J Kidney Dis 1989;13:29-34.

4. Mogensen CE. Prediction of clinical diabetic nephropathy in IDDM patients: alternatives to microalbuminuria? Diabetes 1990;39:761-7.

5. Giampietro O, Clerico A. Microalbuminuria in diabetes: which method to employ, which sample to collect. J Nucl Med Allied Sci 1990;34:111-20.

6. Rowe DJ, Dawnay A, Watts GF. Microalbuminuria in diabetes mellitus: $r \theta-$ view and recommendations for the measurement of albumin in urine. Ann Clin Biochem 1990;27:297-312.

7. Pilo A, Zucchelli M, Piro MA. External quality control for T3 and T4 assays in 
Italy: outline of the scheme and results of the first semester experience. $J$ Nucl Med Allied Sci 1981;25:107-18.

8. Zucchelli GC, Pilo A, Chiesa MR, Piro MA. Progrees report on a national qualitycontrol survey of triiodothyronine and thyroxin assay. Clin Chem 1984;30:397-8.

9. Pilo A, Zucchelli GC, Chiesa MR. Components of variance analysis for data collected in interlaboratory quality control studies of immunoassay. J Nucl Med Allied Sci 1985;29:269-73.

10. Horwitz W. Protocol for the design, conduct and interpretation of collaborative studies. Pure Appl Chem 1988;60:855-64. 11. Giampietro O, Clerico A, Cruschelli L, et al. Microalbuminuria in diabetes mellitus: more on urine storage and accuracy of colorimetric assays [Letter]. Clin Chem 1989;35:1560-2.

12. Giampietro O, Miccoli R, Clerico A, et al. Urinary albumin excretion in normal subjects and in diabetic patients measured by radioimmunoessay: methodological and clinical aspects. Clin Biochem 1988;21:63-8.

13. Mueller PW, MacNeil ML, Smith SJ, Miller DT. Interlaboratory comparison of the measurement of albumin in urine. Clin Chem 1991;37:191-5.

\section{Giuseppe Penno' Aldo Clerico' Lorella Cruschelli Giusy Rigamonti Gaetano Crepaldi ${ }^{3,4}$ Renzo Navalesi ${ }^{1}$ Ottavio Giampietro ${ }^{1,5}$}

${ }^{1}$ Cattedra di Malattie del Metab.

Istituto di Clin. Medica II and Istituto di Fisiol. Clin. del CNR.

University of Pisa

Pisa, Italy

${ }^{2}$ ICI Pharma

Milano, Italy

${ }^{3}$ Istituto di Med. Interna e di Med. Clin.

University of Padua

Padua, Italy

- Study Group Coordinator, Italian Microalbuminuria Study Group.

${ }^{5}$ Address correspondence to this author, at: Cattedra di Malattie del Metabolismo, Istituto di Clinica Medica II Via Roma 67, 56100 Pisa, Italy.

\section{Monitoring Nutritional Status in Burn Patlents}

\section{To the Editor:}

Rettmer et al. (1) compared the performance of several static measurements of serum concentrations with some functional tests for the nutritional assessment of burn patients. Although their conclusion that functional tests generally provide a more accurate evaluation of nutritional sta- tus is probably correct, their interpretation of their results is questionable. To compare these two categories of tests, the nutritional status of the studied patients must be known. The authors classified all their patients as nutritionally replenished, this judgment being based on the estimated calories met, the weight change, and the nitrogen balance. However, these three characteristics reflect only the protein and caloric nutritional status. They do not necessarily indicate that concentrations of all trace minerals and vitamins are normal. Furthermore, these measures are also limited as markers of protein-calorie status in burn patients $(2,3)$. For example, one of their patients achieved only $46 \%$ of his estimated calorie needs but was nonetheless considered to have been adequately fed. Weight gain is difficult to interpret in burn patients because they can develop considerable edema, which accounts for the greater part of the weight gain in the first weeks after the burn. In calculating the nitrogen balance, Rettmer et al. did not account for the losses of protein in burn wounds, which can amount to several grams per day (4).

The authors did not mention the composition of the diet. There is great diversity in the practices of burn centers concerning supplementation with trace minerals and vitamins $(5,6)$. Because Rettmer et al. did not evaluate the same nutrients with the static and the functional tests, we cannot be sure whether the differences they observed only reflect an insufficient supplementation of some elements.

The choice of some of the static tests is also dubious. Even in ambulatory patients, no one would judge the nutritional status by measuring singly the serum concentration of calcium or iron. A low serum concentration of calcium must be interpreted in light of the albumin concentration (7). Similarly, the concentration of transferrin (or the binding capacity of iron) must be known before one can interpret a low serum iron concentration. Also, the units for transferrin in their Figure 1 should read $\mathrm{g} / \mathrm{L}$ instead of $\mathrm{mg} / \mathrm{L}$. If we take into account the low concentration of binding proteins, the observed concentrations of iron and calcium are probably in the low normal range. The same phenomenon could explain the "low" serum copper values obtained (8).

The serum concentration of carotene can reflect the nutritional status of vitamin A only if it is a major source in the diet (9). Because their patients were probably supplemented with the vitamin and not the provitamin (caro- tene), the low concentrations obtained should have been expected.

Finally, their Figure 4 contains several errors. The results of the functional assays are presented in the form of activity coefficients, which are dimensionless. The units indicated $(\mu \mathrm{mol} / \mathrm{L})$ are thus inappropriate. The range of the zinc protoporphyrin/heme ratio cannot be correct because no results below the mean are indicated.

Despite the above restrictions, I agree with Rettmer et al. that there is a great need to develop new nutritional-assessment procedures.

\section{References}

1. Rettmer RL, Williamson JC, Labbe RF, Heimbach DM. Laboratory monitoring of nutritional status in burn patients. Clin Chem 1992;38:334-7.

2. Pasulka PS, Wachtel TL. Nutritional considerations for the burned patient. Surg Clin North Am 1987;67:109-31.

3. Jensen TG, Long JM, Dudrick SJ, Johnston DA. Nutritional aseesement indications of postburn complications. J Am Diet Assoc 1985;85:68-72

4. Waxman K, Rebello T, Pinderski L, et al. Protein lose acroes burn wounds. J Trauma 1987;27:136-40.

5. Shippee RL, Wilson SW, King N. Trace mineral supplementation of burn patients: a national survey. J Am Diet Assoc 1987; 87:300-3.

6. King N, Goodwin CW. Use of vitamin supplements for burned patients: a national survey. J Am Diet Aseoc 1984;84:923-5.

7. Gray TA, Paterson CR. The clinical value of ionised calcium assays. Ann Clin Biochem 1988;25:210-9.

8. Boosalis MG, McCall JT, Solem LD, et al. Serum copper and ceruloplasmin levels and urinary copper excretion in thermal injury. Am J Clin Nutr 1986;44:899-906. 9. Olson JA. Serum levels of vitamin $A$ and carotenoids as reflectors of nutritional status. J Natl Cancer Inst 1984;73:1439-44.

\section{J. Masgé}

Dépt. de Biochim.

Hópital du Saint-Sacrement

Québec, Canada, G1S 4L8

The authors of the article referred to comment:

\section{To the Editor:}

The comments of Masse underscore the difficulty in determining the nutritional status of burn patients (1). The nutritional support our patients received from day 1 post-burn consisted of enteral feeding with one of two standard $1 \mathrm{kcal} / \mathrm{L}$ formulas supplemented by central venous alimentation and a standard multivitamin package if needed. By day 5 all patients were meeting $\geq 84 \%$ of their estimated requirements. By day 15 , 